\title{
Multipath Effect on Covariance Based MIMO Radar Beampattern Design
}

\author{
Amirsadegh Roshanzamir \\ ${ }^{1}$ (Amirkabir university of technology)
}

\begin{abstract}
In a Multiple Input Multiple Output (MIMO) radar unlike conventional phased array radar, we can choose freely between the probing signals transmitted via its antennas to maximize the transmitted power around specific target locations or to approximate a desire beam-pattern in different manners that many papers have taken into consideration so far. In an operational radar or communication environment, one of the usual phenomenon that happens is multipath. In this paper we'd like to take this effect into consideration and show that how this effect can influence our beam-pattern in a covariance based MIMO radar beam-forming. We will show that multipath can change beam-pattern peak direction, increase side-lobe level and either produce false peaks in our desire beam-pattern. MATLAB software is being employed for simulations.
\end{abstract}

Keywords: - MIMO radar, MIMO communication, beam forming, covariance based method.

\section{INTRODUCTION}

Multiple Input Multiple Output (MIMO) radars have attracted many researchers in all over the world recently. This is because of more degrees of freedom in this type of radar which possible choosing freely between probing transmitted signals. This degrees of freedom causes many advantages in this type of radars related to conventional phased array radars [1]-[14].

Generally MIMO radar systems can be classified into two main categories from their antenna placement:

Widely separated MIMO radar[16]

Colocated MIMO radar[17]

In the widely separated case, the antennas are far from each other such that each of them views different aspect of targets radar cross section (RCS) and they will have RCS diversity gain [15]. In colocated antenna, the antennas are close to each other such that all of them views identical aspects of targets being seen $[1],[4]$.

In the other hand many papers have studied MIMO radars wave-forming so far [2]-[13]. From these papers it is understood that MIMO radar wave-forming problem can be broken into three main categories.

Covariance based methods [2]-[6].

Radar ambiguity function based design [7]-[10].

Extended target based design [11]-[13].

In the covariance based method design, cross correlation matric of transmitted signals is designed instead of signals directly. This method has less complexity rather than the other approaches. In the ambiguity function based design cases, entire waveforms will be designed directly to form a desire ambiguity function. And finally in the extended target design method cases like the ambiguity function design method, the entire waveforms are going to design but unlike the ambiguity function methods that considered only point targets, this method include extended targets too [1]. In MIMO radars one can choose freely between the probing signals transmitted via its antennas to maximize the transmitted power around specific target locations or to approximate a desire beam-pattern in different manners that many papers have taken into consideration. The transmitted signals can be all correlated with each other like conventional phased arrays or they can be orthogonal to each other. In an operational radar or communication environment, one of the usual phenomenon that happens is multipath that other papers didn't into consideration. In this paper we'd like to take this effect into consideration and show that how this effect can influence our beam-pattern in a covariance based MIMO radar beam-forming.

The present study has five sections as follows;

section I presents a brief introduction of MIMO radars. In section II we review the covariance based MIMO radar beamforming. Utilization a model of MIMO radar covariance based waveforming in presence of multipath are discussed in section III. Analyzing of multipath effect on beam-pattern peak location and producing false 
peaks related to change in phase variance which inclined by false paths are provided in section IV. Section V is focused on conclusion and references are presented at the end of the paper.

\section{COVARIANCEBASEDMIMORADARS}

Assume that we have a collection of $\mathrm{N}$ transmitter antenna which are located at known coordinates $\boldsymbol{x}_{\boldsymbol{i}}=\left(x_{1, i}, x_{2, i}, x_{3 i}\right)=(x, y, z)$ in some spherical coordinate along the $\mathrm{z}$-axis. In the presented study and in all of the examples and formulas of current paper, it is assume that these transmitter antennas are along the $\mathrm{z}$-axis. It is assume that each transmitter antenna is driven by a specific signal on the carrier frequency of $f_{c}$ or with wavelength of $\lambda$ and complex envelope of $s_{i}(t), i=1, \ldots, N$. At a specific point in space at a distance of $r$ and direction of $\mathrm{k}(\theta, \phi)$ from the transmitter's antenna, each radiated signal $\mathrm{s}_{\mathrm{i}}(\mathrm{t})$ gives rise to a "signal" the far field at radius $\mathrm{r}$, with complex envelope given by

$$
y_{i}(t, r, \theta, \phi)=\frac{1}{\sqrt{4 \pi} r} s_{i}\left(t-\frac{r}{c}\right) e^{j\left(\frac{2 \pi}{\lambda}\right) x_{i}^{T} k(\theta, \phi)}
$$

Where, in this equation, $\mathrm{k}$ is a unit vector in the $(\theta, \phi)$ direction.

At the far field, these signals add linearly and the radiated powers $P_{i}$ add linearly as well. At this point assume that the $\mathrm{i}$-th element location is on the $\mathrm{z}$-axis at coordinate $z_{\mathrm{i}}$. The signal at position $(r, \theta, \phi)$ resulting from all of the transmitted signals at far field will be:

$$
y(t, r, \theta, \phi)=\sum_{i=1}^{N} y_{i}(t, r, \theta, \phi)=\frac{1}{\sqrt{4 \pi}} \sum_{i=1}^{N} s_{i}\left(t-\frac{r}{c}\right) e^{j\left(\frac{2 \pi z_{j}}{\lambda}\right) \sin (\theta \theta)}
$$

The power density is of the entire signals then given by

$$
\mathrm{P}_{\mathrm{y}}(\mathrm{r}, \theta, \phi)=\frac{1}{4 \pi \mathrm{r}^{2}} \sum_{\mathrm{k}=1}^{\mathrm{N}} \sum_{\mathrm{l}=1}^{\mathrm{N}}<\mathrm{s}_{\mathrm{k}}(\mathrm{t}) \mathrm{s}_{\mathrm{l}}^{*}(\mathrm{t})>\mathrm{e}^{\mathrm{j}\left(\frac{2 \pi\left(\mathrm{z}_{\mathrm{k}}-\mathrm{z}_{\mathrm{l}}\right)}{\lambda}\right) \sin (\theta)}
$$

And it is known that the complex signal cross-correlation is defined by

With defining the direction vector as below

$$
\mathrm{R}_{\mathrm{kl}}=\left\langle\mathrm{s}_{\mathrm{k}}(\mathrm{t}) \mathrm{s}_{\mathrm{l}}^{*}(\mathrm{t})\right\rangle
$$

$$
\mathrm{a}(\theta)=\left[\mathrm{e}^{\mathrm{j}\left(\frac{2 \pi z_{1}}{\lambda}\right) \sin (\theta)}, \ldots, \mathrm{e}^{\mathrm{j}\left(\frac{2 \pi z_{\mathrm{N}}}{\lambda}\right) \sin (\theta)}\right]^{\mathrm{T}}
$$

The normalized power density $\mathrm{P}(\theta, \phi)$ of signals, in (W/ster), is

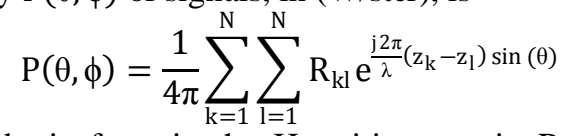

Recognizing that (6) is quadratic form in the Hermitian matrix $\mathrm{R}$ which is the cross-correlation matrix of signals, this can be written compactly as

$$
\mathrm{P}(\theta, \phi)=\frac{1}{4 \pi} \mathrm{a}^{*}(\theta) \operatorname{Ra}(\theta)
$$

This normalized power density $\mathrm{P}(\theta, \phi)$ is exactly the beampattern which we wish to find[3].

First we wish to show some examples of beampatterns produce from such a cross-correlation matrix and further in this paper we will examine effect of multipath on these beam-patterns. Fig. 1 shows the beampattern produced by signal cross-correlation matrix of (8), (9) and (10) respectively (blue, brown and green plots in this figure). It is noticeable that these figures are beam-patterns of 10-element uniform linear array (ULA) with half-wavelength spacing.

$$
\left[\begin{array}{ccc}
1 & \cdots & 1 \\
\vdots & \ddots & \vdots \\
1 & \cdots & 1
\end{array}\right]
$$

$$
\left[\begin{array}{ccc}
0.5^{0} & \cdots & 0.5^{9} \\
\vdots & \ddots & \vdots \\
0.5^{9} & \cdots & 0.5^{0}
\end{array}\right]
$$

$\left[\begin{array}{ccc}0.8^{0} & \cdots & 0.8^{9} \\ \vdots & \ddots & \vdots \\ 0.8^{9} & \cdots & 0.8^{0}\end{array}\right](10)$

In general case the elements of the signal cross-correlation matrix are complex values except the diagonal elements that they are real. This general case is related to MIMO radars but in the case of phased array radar, all 
the transmitter signals are correlated with each other and then all the elements in $R$, are equal to 1(blue one in Fig. 1).

\section{SIGNALMODELINPRESENCEOFMULTIPATH}

In this section we'd like to take multipath into consideration. Then suppose that the transmitted signal from a specific antenna at a desire point in the space with the distance of $r$ and direction of $f(\theta, \phi)$ would be in the following form:

$$
y_{i}(t, r, \theta, \phi)=\sum_{m=1}^{N_{m}} A_{M} \frac{1}{\sqrt{4 \pi} r} s_{i m}\left(t-\frac{r_{m}}{c}\right) e^{j \tau_{i m}}
$$

This is a complete general form for multipath effect. In this equation $N_{m}$ shows the maximum number of paths that the transmitted signals have to receive to desire point it is said maximum number because actually in real, it is possible that different signals come from different paths since we have said here maximum number of paths. $A_{m}$ shows the amplitude of receive signal that in general is a complex value and also can be zero to show that one path doesn't exist for one antenna. $S_{i m}$ shows the m'th path for $i$ 'th antenna, $r_{m}$ denotes the distance of m'th path and $\tau_{i m}$ is the phase shift which occurs during m'th path to i'th antenna. This equation has many unknown parameters and then working with that is complex, so for the following we take bellow simplifier assumption into consideration.

Each multipath occurs from a path that have almost the distance of line of sight path. It means that $r_{m}=r$. Actually this occurs when the obstacles which cause the multipath be close to transmitter antennas

With the above assumption we can write (1) in the following way:

$$
\mathrm{y}_{\mathrm{i}}(\mathrm{t}, \mathrm{r}, \theta, \phi)=\sum_{\mathrm{m}=1}^{\mathrm{N}_{\mathrm{m}}} \mathrm{A}_{\mathrm{M}} \frac{1}{\sqrt{4 \pi} \mathrm{r}} \mathrm{s}_{\mathrm{im}}\left(\mathrm{t}-\frac{\mathrm{r}}{\mathrm{c}}\right) \mathrm{e}^{\mathrm{j} \tau_{\mathrm{im}}}
$$

Since we take $A_{m}$ complex value, we eliminate dependence of phase shift to multipath and include it's influence into $A_{m}$ then (12) equation can be written as

$$
y_{i}(t, r, \theta, \phi)=\sum_{m=1}^{N_{m}} A_{M} \frac{1}{\sqrt{4 \pi} r} s_{i m}\left(t-\frac{r}{c}\right) e^{j \tau_{i}}
$$

It is noticeable that we take

$$
A_{m}=1, m=1
$$

Which shows the normalize amplitude for line of sight signal.

Now with the above expression, sum of the all the transmitted signals with their multipath can be written as follow:

$$
y(t, r, \theta, \phi)=\sum_{i=1}^{N} y_{i}(t, r, \theta, \phi)=\frac{1}{\sqrt{4 \pi} r} \sum_{m=1}^{N_{m}} \sum_{i=1}^{N} A_{m} s_{i}\left(t-\frac{r}{c}\right) e^{j \tau_{i}}
$$

The power density is of the entire signals then given by

$$
P_{y}(r, \theta, \phi)=\frac{1}{4 \pi r^{2}} \sum_{j=1}^{N_{m}} \sum_{m=1}^{N_{m}} \sum_{k=1}^{N} \sum_{l=1}^{N}<A_{m} s_{k}(t) A_{j}^{*} s_{l}^{*}(t)>e^{j\left(\tau_{k}-\tau_{l}\right)}
$$

That can be written as follow:

$$
P_{y}(r, \theta, \phi)=\frac{1}{4 \pi r^{2}} \sum_{j=1}^{N_{m}} A_{j}^{*} \sum_{m=1}^{N_{m}} A_{m} \sum_{k=1}^{N} \sum_{l=1}^{N}<s_{k}(t) s_{l}^{*}(t)>e^{j\left(\tau_{k}-\tau_{l}\right)}
$$

Then the normalized power density $\mathrm{P}(\theta, \phi)$ of signals, in (W/ster), is

$$
\mathrm{P}(\theta, \phi)=\frac{1}{4 \pi} \sum_{\mathrm{j}=1}^{\mathrm{N}_{\mathrm{m}}} \mathrm{A}_{\mathrm{j}}^{*} \sum_{\mathrm{m}=1}^{\mathrm{N}_{\mathrm{m}}} \mathrm{A}_{\mathrm{m}} \sum_{\mathrm{k}=1}^{\mathrm{N}} \sum_{\mathrm{l}=1}^{\mathrm{N}} \mathrm{R}_{\mathrm{kl}} \mathrm{e}^{\mathrm{j}\left(\tau_{\mathrm{k}}-\tau_{\mathrm{l}}\right)}
$$

Just like above recognizing that (18) is quadratic form in the Hermitian matrix R which is the cross-correlation matrix of signals, this can be written compactly as

$$
P(\theta, \phi)=\frac{1}{4 \pi} \sum_{j=1}^{N_{m}} A_{j}^{*} \sum_{m=1}^{N_{m}} A_{m}\left[a^{*}(\theta) \operatorname{Ra}(\theta)\right]
$$


So this expression denotes beam-pattern in presence of multipath effect. In next section with some numerical examples we investigate this effect and its influence on the desire beam-pattern.

\section{NUMERICALEXAMPLES}

In this section we wish to consider effect of multipath on our desire beam-pattern. We will examine this effect in two manners. First we would like to investigate this effect on location of target or directions that we wish to have peak in our beam-pattern, second we'd like to examine effect of multipath on amplitude of beampattern on desired directions and false peaks that occur during this effect. In the following we will examine these cases.

\section{A. Multipath effect on phased array}

As it stated on previous section for a phased array radar we deal with a matric cross correlation of signals which all the elements are equal to one. Fig. 1 shows multipath effect on such a case.

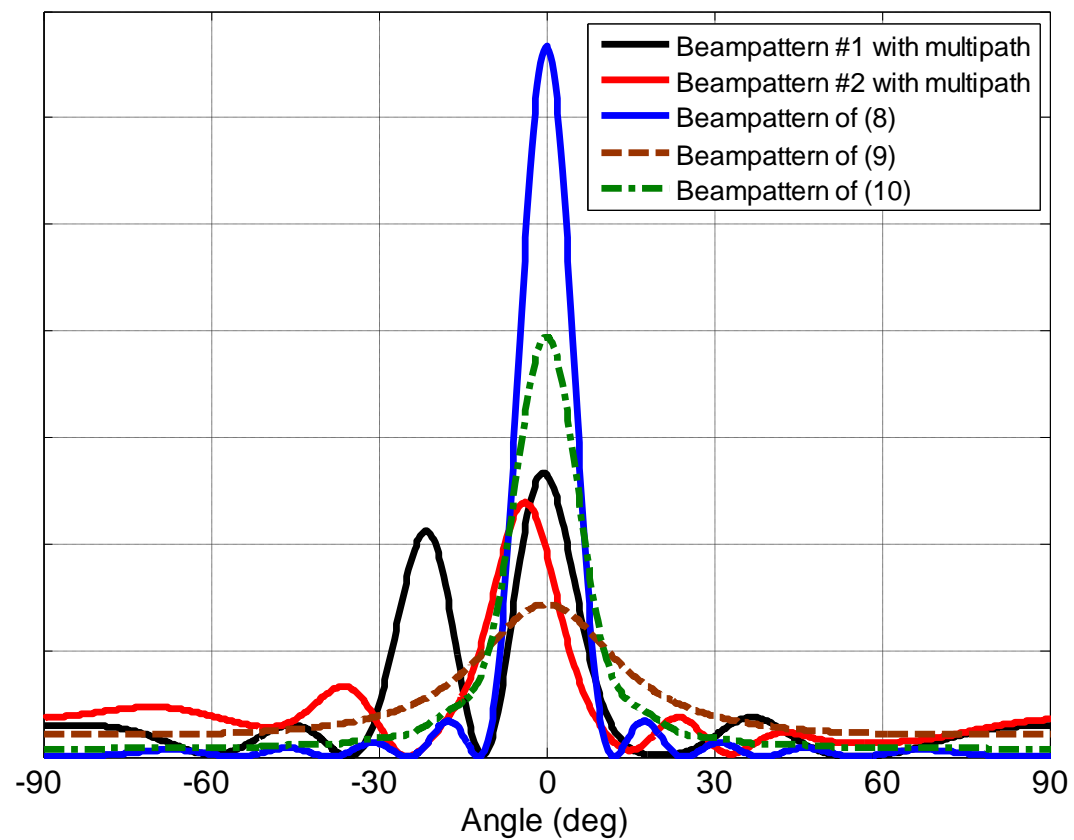

Fig. 1.Beam-pattern of phased array respect to (7). The blue one is corresponds to phases array without any multipath and black one and red one is related to phased array pattern with multipath. Brown and green plots are corespond to (9) and (10) expressions respepectly

This figure depicts all we need. First of all it should mention that we consider two assumption in this figure. First we assume that each signal has two path to desire point of interest in other words one path is line of sight and our desire path, and there is only one another path for each signal to that point of interest amplitude of false path have taken equal to line of sight case and it inclined only a random phase with normal distribution with zero mean and $\pi$ radians variance to signal of false path. Second assumption is that we take the total power at the point of interest identical for both multipath case and without multipath case. It is noticeable that these figures are beam-patterns of 10-element uniform linear array (ULA) with half-wavelength spacing. As it seen from this figure, in both cases of multipath side-lobe levels have increase related to phased array case without multipath. Multipath can also change the exact location of beam-pattern peak from our desire direction, the case which has occurred due to red plot in Fig. 1 and at the other hand as it is seen from black plot from this figure it can cause false peaks in our beam-pattern. In next sections we will consider this effect in more details.

\section{B. Random phase and amplitude effect on peak location of desire beampattern}

In this section we wish to consider that how multipath with different number of paths, random amplitude and random phase can affect our desire beam-pattern in direction, amplitude (side-lobe level) and false peaks. In this section and following sections we will take our desire beam-pattern just like the case shown in Fig. 1 which is in green color. Since it has one peak and has no side-lobe we can examine multipath effect more easily and more accurate.

In Fig. 2 we have considered the case that there is only two paths from each transmitter antenna to a desire point of interest which one of them is line of sight and our desire path and one of them is false path. and 
also we have assume that the false path is inclined the signals with random amplitude of uniform distribution between 0 and 1 ( 1 is normalized amplitude for the line of sight signal amplitude) and random phase with normal distribution with zero mean and variance of between 0 and $\pi$ radians. Then we wish to consider mean error of peak power direction of the beam-pattern refer to change in phase variance between 0 and $\pi$ radians.

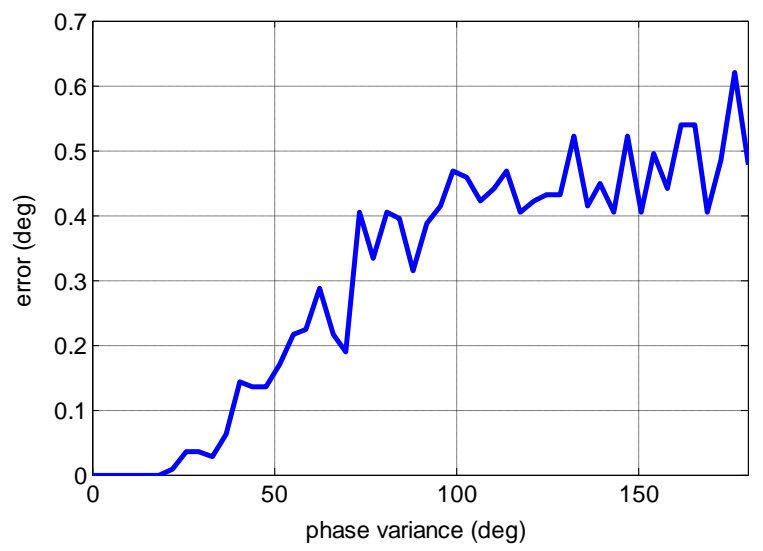

Fig. 2. mean error of beam-pattern peak direction in presence one path of multipath

For a special case, in Fig. 3 we have shown mean error of above case but with signal amplitude of false path equal to 1 . That means equal to amplitude of line of sight signal. Both of these plots have drawn for 300 iterations in each point.

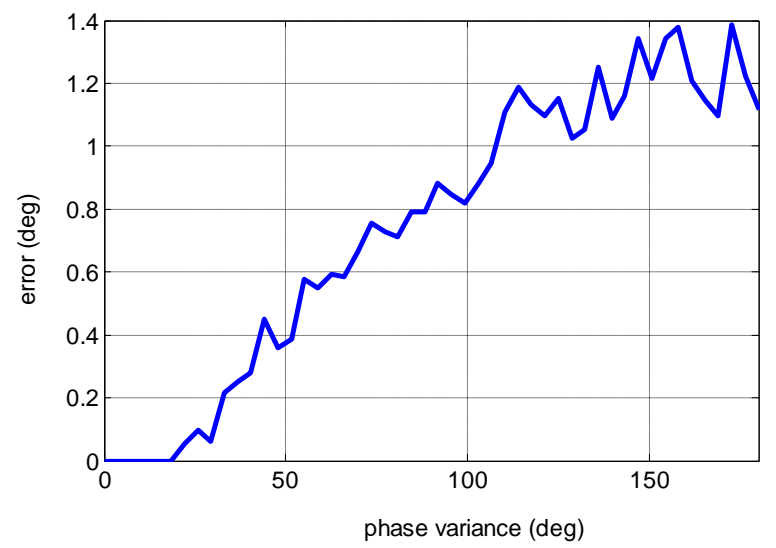

Fig. 3. mean error of beam-pattern peak direction in presence of one path of multipath with constant amplitude of multipath signal

From these figures it is apparent that as phase variance of multipath signal increase error of beampattern peak direction will increase as well. This is just done for amplitude of signal which is obtained by comparing Fig. 2 and 3 . As it is seen from these figures with constant amplitude equal to line of sight signal, this figure has two times bigger error in estimating the exact angle to focusing power.

\section{Effect of number of path in multipath on peak location of desire beam-pattern}

In this section we are interested in examine the effect of number of paths to our previous section result. For this purpose we consider the case which there is 10 elements half wave length uniform array just like the previous section. We also consider the case which all false paths inclined random amplitude with uniform distribution between 0 to 1 ( 1 is one for the line of sight path) and random phase with normal distribution of zero mean and variable variance between 0 to $\pi$, to the transmitted signals. But here we consider three false paths instead of one false path in previous section. In other words in this case including line of sight path, totally we have four paths to desire point of interest. Like the previous section, Fig. 4 is related to general case with random amplitude of multipath signal and Fig. 5 is related to specific case which all the false paths have identical amplitude equal to one for line of sight signal. That means actually they are counterparts of Fig. 2 and 3 with three false paths. 


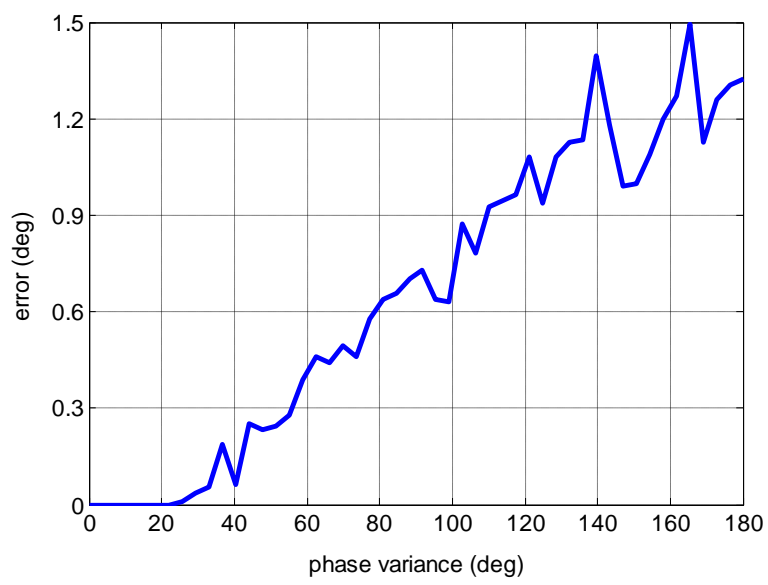

Fig. 4.mean error of beam-pattern peak direction in presence of three path of multipath

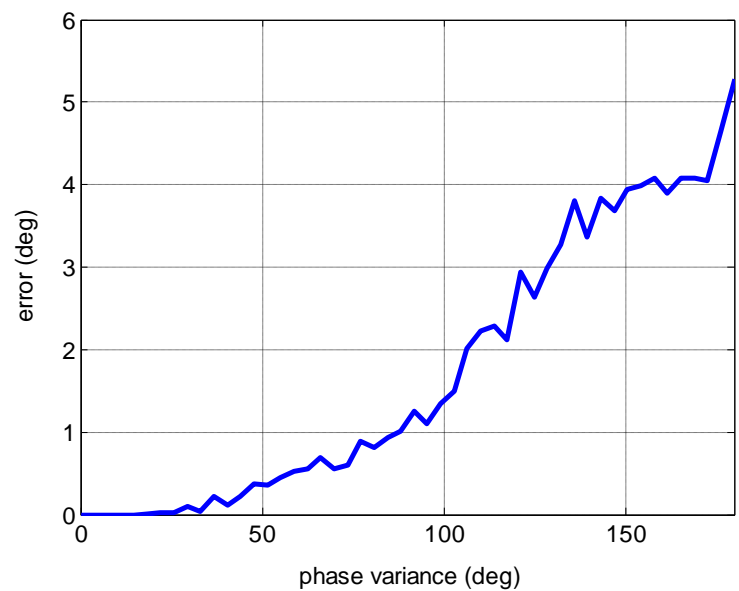

Fig. 5. mean error of beam-pattern peak direction in presence of three path of multipath with constant amplitude of multipath signal

As it seen from these figures error in focusing the beam-pattern to a desire direction will increase as the paths of multipath increase. This is an important parameter in operational environment that how many source of multipath we may have. Answer to this question will help us to use suitable signals to decrease influence of multipath and achieve our goal. Fig. 6 shows mean error in beam-pattern peak direction related to change into number of multipath paths. For this figure we have taken paths of multipath amplitudes random with uniform distribution between 0 and 1 ( 1 means normalize value of line of sight amplitude signal) and the phase which these paths inclined to signals has taken random variables with normal distribution of zero mean and variance of $\pi$. This figure has plotted for 1 to 10 number of false paths.

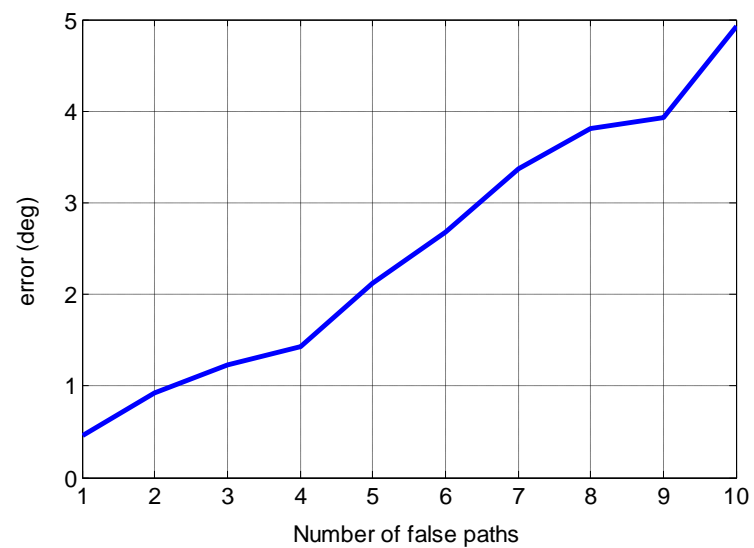

Fig. 6. mean error of beam-pattern peak direction in presence of multipath related to number of false paths 
As it seen from this figure as number of false paths increase, mean error of beam-pattern peak location will increase as well.

In this section we investigated effect of multipath to beam-pattern peak direction. At next section we will consider effect of multipath on false peak that produce due to this effect.

\section{Multipath effect on false peaks}

In this section we will investigate multipath effect on total shape of beam-pattern and we will examine this effect on producing false peaks that may occur during this effect as it shown previously in Fig. 1. like latter sections, in this section we ignore power difference at the points of interest due to multipath. For comparison reasons we will take total power at desire points all identical. In here we consider the case which there is 10 elements half wave length uniform array just like the previous sections. We also consider the case which all false paths inclined random amplitude with uniform distribution between 0 to 1 ( 1 is one for the line of sight path) and random phase with normal distribution of zero mean and variable variance between 0 to $\pi$, to the transmitted signals. As in this section we are looking for false peaks, we have to define a standard to recognizing false peaks. We define this standard as be at least half of the main beam. In the other words, if one side-lobe be at least as bigger as half of the main beam amplitude, we name this side-lobe as false peak.

\section{E. Random phase and amplitude effect on false peaks}

In this section we wish to consider that how multipath with random amplitude and random phase can affect our desire beam-pattern and producing false peaks. In Fig. 7 we have considered the case that there is only two paths from each transmitter antenna to a desire point of interest which one of them is line of sight and our desire path and one of them is false path, and also we have assume that the false path is inclined the signals with random amplitude of uniform distribution between 0 and 1 ( 1 is normalized amplitude for the line of sight signal amplitude) and random phase with normal distribution with zero mean and variance of between 0 and $\pi$ radians. Then we wish to see in how many cases of our examination multipath produces at least one false peak related to change in phase variance between 0 and $\pi$ radians.

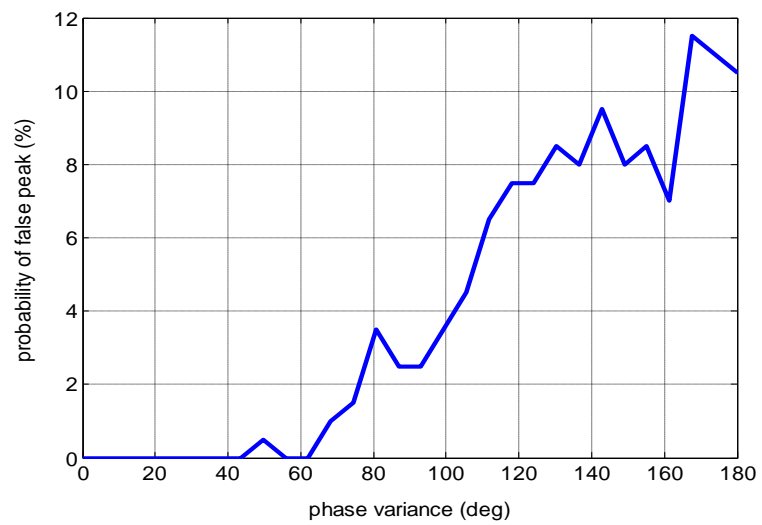

Fig. 7. mean error of beam-pattern peak direction in presence one path of multipath

For a special case, in Fig. 8 we have shown percentage of error of above case but with signal amplitude of false path equal to 1 . That means equal to amplitude of line of sight signal. Both of these plots have drawn for 300 iterations in each point.

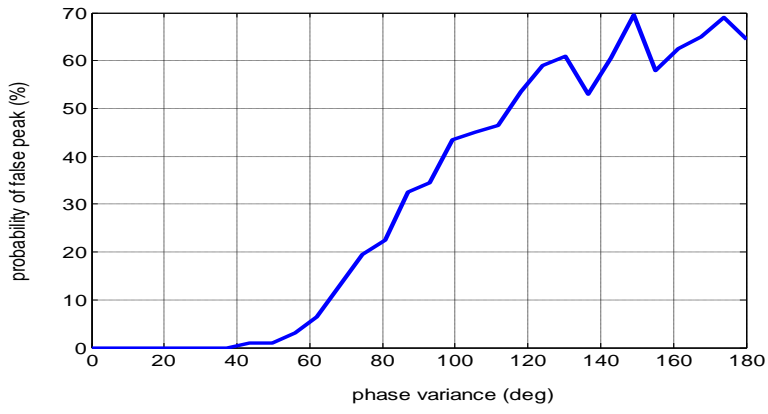

Fig. 8. mean error of beam-pattern peak direction in presence of one path of multipath with constant amplitude of multipath signal 
From these figures it is obvious that as phase variance of multipath signal increase percentage of having false peak will increase as well. This is just done for amplitude of signal which is obtained by comparing Fig. 7 and 8 . As it is seen from these figures with constant amplitude equal to line of sight signal, this figure has five times bigger error in estimating the exact angle to focusing power.

\section{F. Effect of number of paths in multipath on false peaks of desire beam-pattern}

In this section we are interested in examine the effect of number of paths to our previous section results. For this purpose we consider the case which there is 10 elements half wave length uniform array just like the previous sections. We also consider the case which all false paths inclined random amplitude with uniform distribution between 0 to 1 ( 1 is one for the line of sight path) and random phase with normal distribution of zero mean and variable variance between 0 to $\pi$, to the transmitted signals. But here we consider three false paths instead of one false path in previous section. In other words in this case including line of sight path, totally we have four paths to desire point of interest. Like the previous section, Fig. 9 is related to general case with random amplitude of multipath signal and Fig. 10 is related to specific case which all the false paths have identical amplitude equal to one for line of sight signal. That means actually they are counterparts of Fig. 7 and 8 with three false paths.

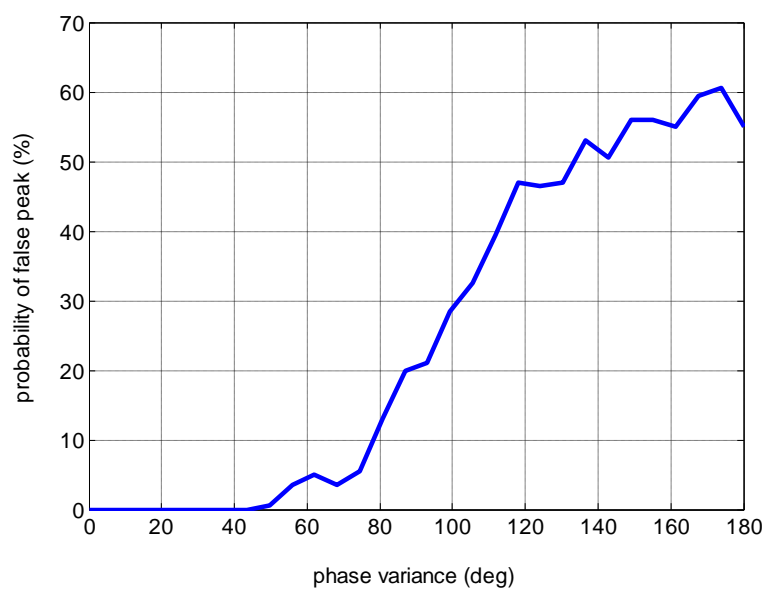

Fig. 9.mean error of beam-pattern peak direction in presence of three path of multipath

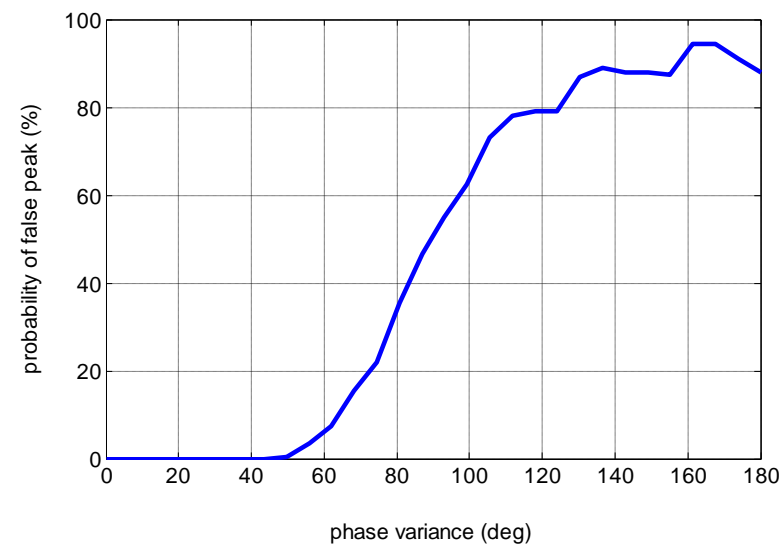

Fig. 10. mean error of beam-pattern peak direction in presence of three path of multipath with constant amplitude of multipath signal

As it seen from these figures, false peak error probability will increase as the paths of multipath increases. This is an important parameter in operational environment that how many source of multipath we may have. Answer of this question will help us to use suitable signals to decrease influence of multipath and achieve our goal. Fig. 11 shows false peak error probability in beam-pattern related to change into number of multipath paths. For this figure we have taken paths of multipath amplitudes random with uniform distribution between 0 and 1(1 means normalize value of line of sight amplitude signal) and the phase which these paths inclined to 
signals has taken random variables with normal distribution of zero mean and variance of $\pi$. This figure has plotted for 1 to 10 number of false paths and for iteration of 300 times .

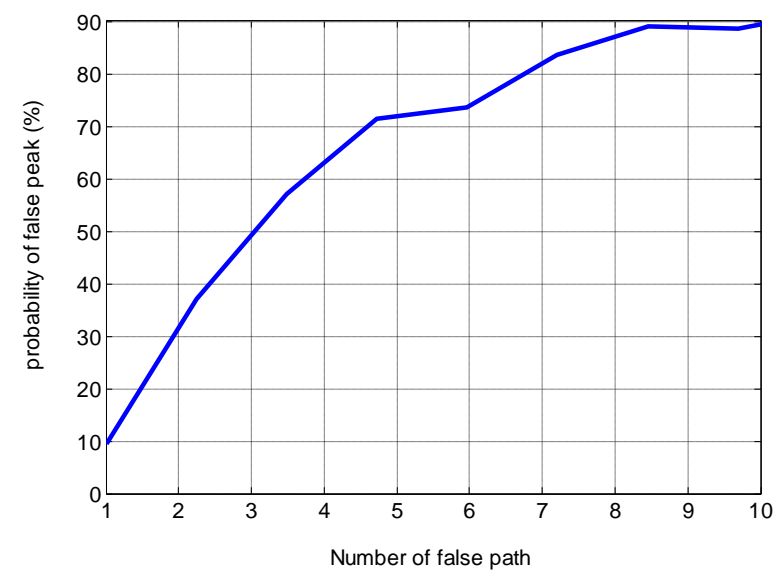

Fig. 11. mean error of beam-pattern peak direction in presence of multipath related to number of false paths

As it seen from this figure as number of false paths increase, false peak error probability of beam-pattern will increase as well.

In this section we investigated effect of multipath to beam-pattern false peak probability.

\section{CONCLUSIONANDFUTUREWORKS}

At the present study we have shown that multipath effect on our desire beam-pattern. And it is shown that multipath can change the desire beam-pattern peak location, increase side-lobe level and produce false peaks. The result that have provided in this paper can be used to selecting suitable waveforms with suitable cross correlation matric to reduce these effects, and further more we can have an estimation of intensity of multipath effect in different operational environments. As it said earlier in this paper for dealing more easily with multipath, we ignore time delay difference from multiple paths in our multipath effect model. As a future work one can take this effect into consideration too and solve these problems without that assumption again. Also as it presented, we took uniform distribution for amplitude of signal and normal distribution with zero mean for phase of its which inclined by multipath on transmitted signals. one can take another model for amplitude and phase of signals which pass throw false paths as a future work.

\section{REFERENCES}

[1] Chun-Yang Chen, "MIMO Radar Waveform Optimization With Prior Information of the Extended Target and Clutter" Signal Processing, IEEE Transaction Vol. 57 , Issue: 9 pp. 3533 - 3544 Sept. 2009.

[2] D. R. Fuhrmann and G. S. Antonio, "Transmit beamforming for MIMO radar systems using partial signal correlation," in Proc. 38th IEEE Asilomar Conf. Signals, Syst., Comput., Nov. 2004, pp. 295-299.

[3] D. R. Fuhrmann and G. S. Antonio, "Transmit beamforming for MIMO radar systems using signal crosscorrelation,” IEEE Trans. Aerosp. Electron. Syst., vol. 44, pp. 171-186, Jan. 2008.

[4] P. Stoica, J. Li, and Y. Xie, "On probing signal design for MIMO radar," IEEE Trans. Signal Process., vol. 55, no. 8, pp. 4151-4161, Aug. 2007.

[5] J. Li, L. Xu, P. Stoica, K. W. Forsythe, and D. Bliss, "Range compression and waveform optimization for MIMO radar: A Cramér-Rao bound based study," IEEE Trans. Signal Process., vol. 56, no. 1, pp. 218 232, Jan. 2008.

[6] P. Stoica, J. Li, and X. Zhu, "Waveform synthesis for diversity-based transmit beampattern design," IEEE Trans. Signal Process., Jun. 2008.

[7] C. Y. Chen and P. P. Vaidyanathan, "MIMO radar ambiguity properties and optimization using frequency-hopping waveforms," IEEE Trans. Signal Process., Dec. 2008.

[8] J. Li, P. Stoica, and X. Zheng, "Signal synthesis and receiver design for MIMO radar imaging," IEEE Trans. Signal Process., Aug. 2008.

[9] D. Kornack and P. Rakic, "Cell Proliferation without Neurogenesis in Adult Primate Neocortex," Science, vol. 294, Dec. 2001, pp. 2127-2130, doi:10.1126/science.1065467.

[10] B. Liu, Z. He, and Q. He, "Optimization of orthogonal discrete frequency-coding waveform based on modified genetic algorithm for MIMO radar," in Proc. Int. Conf. Commun., Circuits, Syst., Jul. 2007, pp. 966-970. 
[11] B. Friedlander, "Waveform design for MIMO radars," IEEE Trans. Aerosp. Electron. Syst., vol. 43, pp. 1227-1238, Jul. 2007.

[12] Y. Yang and R. S. Blum, "MIMO radar waveform design based on mutual information and minimum mean-square error estimation," IEEE Trans. Aerosp. Electron. Syst., vol. 43, no. 1, pp. 330-343, Jan. 2007.

[13] Y. Yang and R. S. Blum, "Minimax robust MIMO radar waveform design," IEEE J. Sel. Topics Signal Process., vol. 1, no. 1, pp. 147-155, Jun. 2007.

[14] E. Fishler, A. Haimovich, R. Blum, L. Cimini, D. Chizhik, and R. Valenzuela, "Performance of MIMO radar systems: Advantages of angular diversity," in Proc. 38th Asilomar Conf. Signals, Syst. Comput., Nov. 2004, vol. 1, pp. 305-309.

[15] E. Fishler, A. Haimovich, R. Blum, D. Chizhik, L. Cimini, and R. Valenzuela, "MIMO radar: An idea whose time has come," in Proc. IEEE Radar Conf., Apr. 2004, pp. 71-78.

[16] A. M. Haimovich, R. S. Blum, and L. J. Cimini, "MIMO radar with widely separated antennas," IEEE Signal Process. Mag., vol. 25, no. 1, pp. 116-129, Jan. 2008.

[17] J. Li and P. Stoica, "MIMO radar with colocated antennas," IEEE Signal Process. Mag., vol. 24, no. 5, Sep. 2007. 